\title{
Thermodynamic Properties of Fluids from Speed of Sound: Integration Along Isentropes
}

\author{
M. Bijedić ${ }^{*}$, E. Đidić ${ }^{2}$ \\ ${ }^{1}$ Faculty of Technology, University of Tuzla, 8 Univerzitetska Str., 75000 Tuzla, Bosnia and Herzegovina \\ ${ }^{2}$ BNT - Factory of Machines and Hydraulics, 1 Mehmeda Spahe Str., 72290 Novi Travnik, Bosnia and Herzegovina \\ E-mail: ${ }^{1}$ muhamed.bijedic@untz.ba ${ }^{2}$ enver.dzidic@bnt-tmh.ba
}

Received 23 August 2021, Revised 25 October 2021, Accepted 1 November 2021

\begin{abstract}
The equations connecting speed of sound with other thermodynamic properties of gases and liquids, suitable for numerical integration with respect to temperature, density, and pressure, along isentropes, are derived. Algorithms of their solution are given too. They are tested with several substances (e.g., $\mathrm{Ar}, \mathrm{N}_{2}, \mathrm{O}_{2}, \mathrm{CH}_{4}, \mathrm{CO}_{2}$, and $\mathrm{H}_{2} \mathrm{O}$ ) in wide ranges of pressure and temperature. Average absolute deviation of thermal properties is $0.0129 \%$ in supercritical gaseous phase, $0.0308 \%$ in transcritical gaseous phase, and $0.0009 \%$ in liquid phase. Corresponding deviations of caloric properties are $0.1706 \%, 0.1863 \%$, and $0.0702 \%$, respectively.
\end{abstract}

\section{Keywords: Thermodynamic properties; fluids; speed of sound, entropy.}

\section{Introduction}

Thermodynamic properties of gases above their critical temperature may be derived from speed of sound if pressure and temperature, or density and temperature, are used as independent variables. In the former case, initial values of dependent variables (e.g., density and isobaric heat capacity) are specified along the lowest temperature at several pressures. In the later case, initial values of dependent variables (e.g., pressure and isochoric heat capacity) are specified along the lowest temperature at several densities. Numerical integration is performed with respect to temperature in both cases, but along isobars and isochores, respectively. When speed of sound is measured in the same pressure range at each temperature, these data are best exploited if integration is performed along isobars [1,2]. For initial values specified in the same pressure range, integration along isochores will cover wider pressure range [3]. In this case, initial values may also be specified along the lowest density (e.g., in the limit of ideal gas) at several temperatures, and integration performed with respect to density along isotherms [4]. However, in order to retain stability of the solution, boundary values are needed along the lowest temperature(s). The same sets of initial values may be used to carry out integration below critical temperature. In this case, pressure [2] and density [3, 5] are divided by their corresponding values at saturation, at each temperature, and these quantities are used as new independent variables instead of pressure and density, respectively.

When it comes to liquid phase above critical pressure, temperature and pressure are used as independent variables. Initial values of dependent variables (e.g., density and isobaric heat capacity) are specified along the lowest pressure at several temperatures. Numerical integration is performed with respect to pressure along isotherms $[6,7]$. The same set of initial values may be used to carry out integration below critical pressure. In this case integration is performed along paths whose shapes gradually change from that of an isotherm to that of the saturation line [6]. Also, initial values may be specified along the lowest pressure at several temperatures and integration performed along the same paths in opposite direction, or along isotherms with temperature range being extended to the saturation line in each integration step [7]. However, in order to retain stability of the solution in two later approaches, boundary values are needed along the saturation line. Initial values may also be specified along the saturation line and integration performed with respect to pressure along isotherms, but with front of integration having shape of the saturation line rather than that of isobar [6].

If all derivatives appearing in equations connecting speed of sound with other thermodynamic properties (of gases and vapors) are expressed in terms of finite differences [8] or cubic splines [9], the sets of nonlinear algebraic equations are obtained. They can be solved for pressure and heat capacity in very wide ranges of temperature and density. Unlike an approach based on numerical integration, which requires initial values not only of thermal but also of caloric properties (or of thermal ones but of Neumann type), this approach requires only boundary values of thermal properties of Dirichlet type. However, they have to be imposed along overall boundary (e.g., along two isotherms and two isochores).

For initial values specified in the same pressure range, integration along isentropes in gaseous phase will cover wider pressure range than integration along isochores, as one can see from example given at Figure 1. Here, temperature and entropy are used as independent variables.

If density and entropy are used as independent variables, domain of integration could be increased even further, as one can see from example given at Figure 2.

Similarly, for initial values specified in the same temperature range, integration along isentropes in liquid phase will cover wider temperature range than integration 
along isotherms, as one can see from example given at Figure 3. Also, isentrope on the left side of integration domain crosses melting line (not seen on the figure) at much higher pressure than corresponding isotherm does. Here, pressure and entropy are used as independent variables.

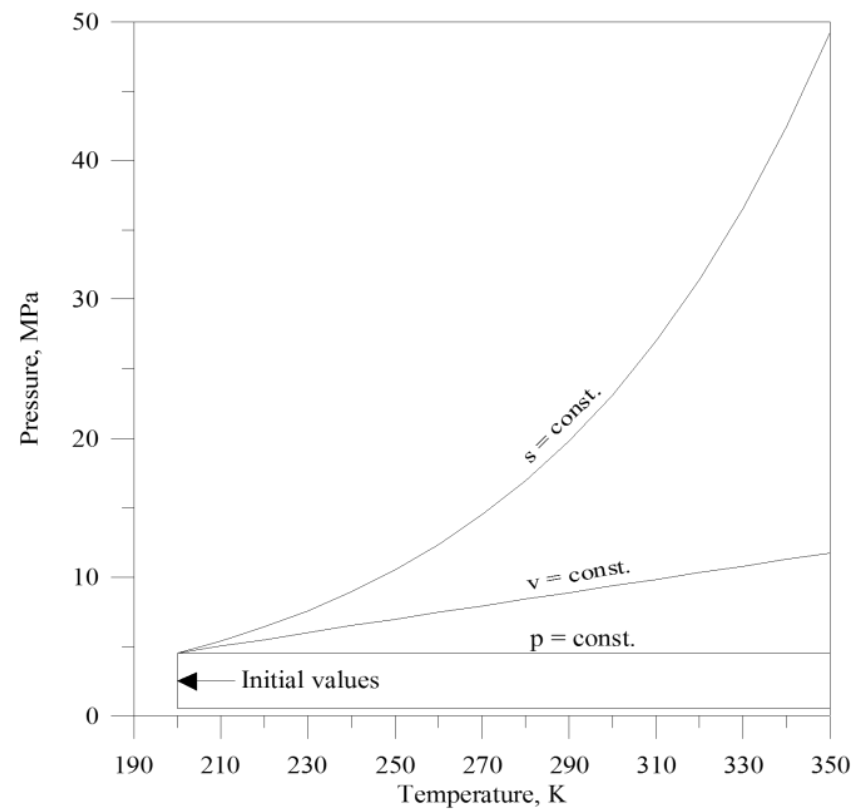

Figure 1. Integration with respect to $T$ at $p=$ const., $v=$ const., and $s=$ const., for methane in gaseous phase [22].

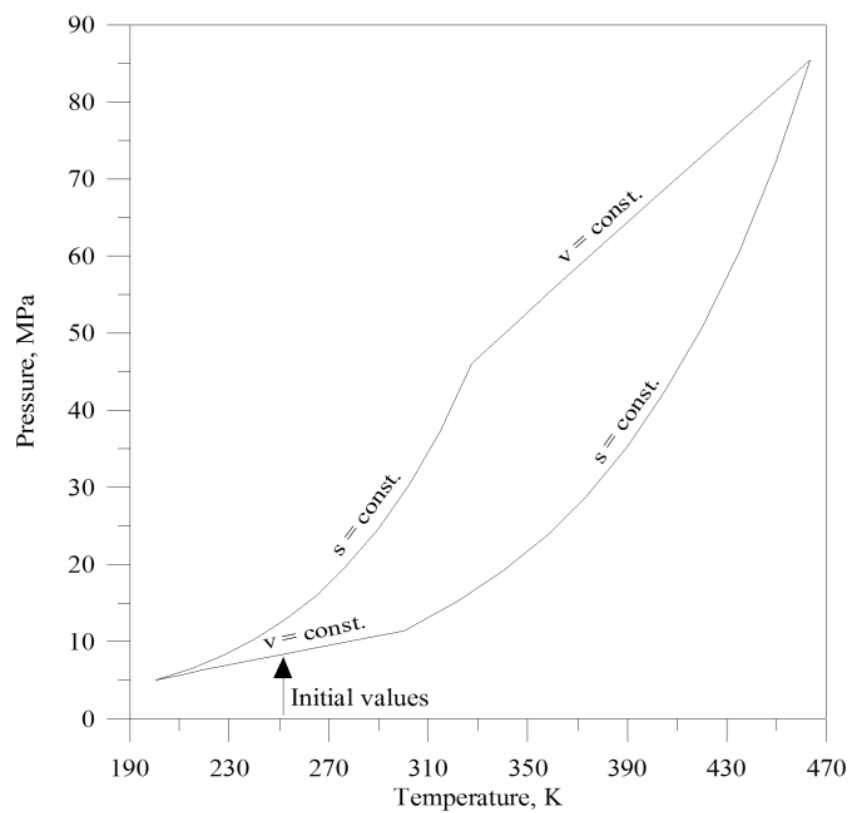

Figure 2. Integration with respect to $\rho$ at $s=$ const., for methane in gaseous phase [22].

Integration with respect to pressure at $T=$ const. in supercritical gaseous phase is not recommended in general, since density increases very quickly with pressure at fixed temperature, and this becomes even more emphasized as critical point is approached. This area of thermodynamic surface could be avoided if integration is conducted at $s=$ const., as one can see from example given at Figure 4.

While entropy is not measurable quantity, it still can be calculated from thermal properties and heat capacity along initial isochore or isobar. Also, it may be used explicitly or implicitly (e.g., in terms of other properties).

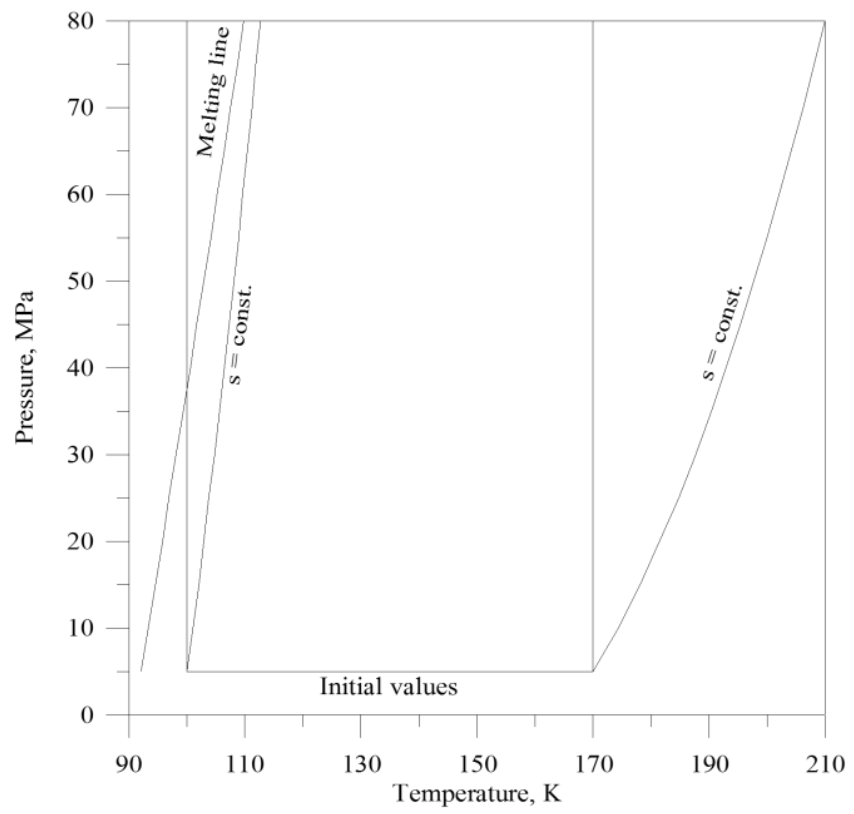

Figure 3. Integration with respect to $p$ at $T=$ const. and $s=$ const., for methane in liquid phase [22].

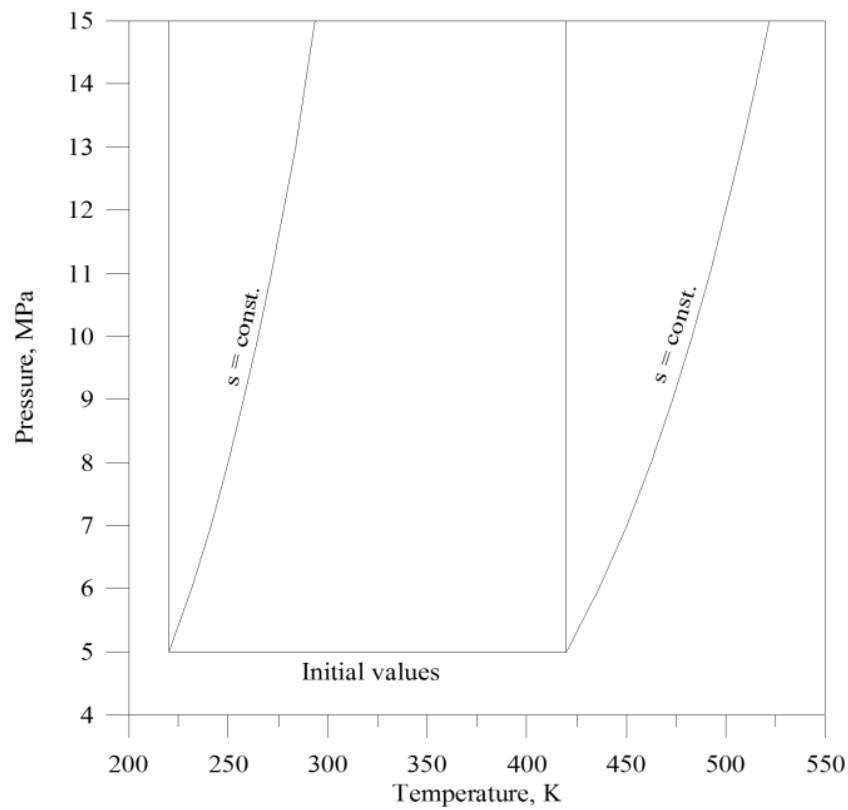

Figure 4. Integration with respect to $p$ at $T=$ const. and $s=$ const., for methane in gaseous phase [22].

\section{Theory}

The speed of sound is an intensive property whose value depends on the state of the medium through which sound propagates. Experiments indicate that the relation between pressure and density across a sound wave is nearly isentropic. The expression for the speed of sound reads [10]

$$
u^{2}=\left(\begin{array}{l}
\partial p \\
\partial \rho
\end{array}\right)_{s}
$$

where: $u$ is the speed of sound, $p$ is the pressure, $\rho$ is the mass density, and $s$ is the specific entropy. However, Eq. (1) may not be solved for $\rho$ since $u$ is not measured along isentropes but rather along isotherms. To overcome this, additional property relations have to be included. 
2.1 Temperature and entropy as independent variables

2.1.1 Isothermal and isochoric derivatives

According to the following rule of differential calculus

$\left(\frac{\partial y}{\partial x}\right)_{a}=\left(\frac{\partial y}{\partial x}\right)_{b}+\left(\frac{\partial y}{\partial b}\right)_{x}\left(\frac{\partial b}{\partial x}\right)_{a}$

one can write

$\left(\frac{\partial p}{\partial T}\right)_{s}=\left(\frac{\partial p}{\partial T}\right)_{\rho}+\left(\frac{\partial p}{\partial \rho}\right)_{T}\left(\frac{\partial \rho}{\partial T}\right)_{s}$

where $T$ is the thermodynamic temperature. According to another rule

$\left(\frac{\partial z}{\partial x}\right)_{a}=\left(\frac{\partial z}{\partial y}\right)_{a}\left(\frac{\partial y}{\partial x}\right)_{a}$

one can also write

$\left(\frac{\partial p}{\partial T}\right)_{s}=\left(\frac{\partial p}{\partial \rho}\right)_{s}\left(\frac{\partial \rho}{\partial T}\right)_{s}$

Combining (1), (3), and (5) one obtains

$$
\begin{aligned}
& \left(\frac{\partial \rho}{\partial T}\right)_{s}=\left(\frac{\partial p}{\partial T}\right)_{\rho}\left[u^{2}-\left(\frac{\partial p}{\partial \rho}\right)_{T}\right]^{-1} \\
& \left(\frac{\partial p}{\partial T}\right)_{s}=u^{2}\left(\frac{\partial \rho}{\partial T}\right)_{s}
\end{aligned}
$$

If rule (2) is applied to the isochoric derivative in (6), it is obtained

$$
\left[\frac{1}{\partial T}\left(\frac{\partial p}{\partial T}\right)_{\rho}\right]_{s}=\left(\frac{\partial^{2} p}{\partial T^{2}}\right)_{\rho}+\frac{\partial^{2} p}{\partial T \partial \rho}\left(\frac{\partial \rho}{\partial T}\right)_{s}
$$

The second order isochoric derivative in (8) may be obtained from the following thermodynamic relation [10]

$$
\left(\frac{\partial^{2} p}{\partial T^{2}}\right)_{\rho}=-\frac{\rho^{2}}{T}\left(\frac{\partial c_{v}}{\partial \rho}\right)_{T}
$$

where $c_{v}$ is the specific heat capacity at constant volume, which may be obtained from

$c_{v}=\frac{T}{\rho^{2}}\left(\frac{\partial p}{\partial T}\right)_{\rho}^{2}\left[u^{2}-\left(\frac{\partial p}{\partial \rho}\right)_{T}\right]^{-1}$

and finally

$c_{p}=c_{v} u^{2}\left(\frac{\partial p}{\partial \rho}\right)_{T}^{-1}$

where $c_{p}$ is the specific heat capacity at constant pressure.
2.1.2 Isothermal and isobaric derivatives

According to the rules (2) and (4) one can also write

$\left(\frac{\partial \rho}{\partial T}\right)_{s}=\left(\frac{\partial \rho}{\partial T}\right)_{p}+\left(\frac{\partial \rho}{\partial p}\right)_{T}\left(\frac{\partial p}{\partial T}\right)_{s}$

$\left(\frac{\partial \rho}{\partial T}\right)_{s}=\left(\frac{\partial \rho}{\partial p}\right)_{s}\left(\frac{\partial p}{\partial T}\right)_{s}$

Combining (1), (12), and (13) one obtains

$\left(\frac{\partial p}{\partial T}\right)_{s}=\left(\frac{\partial \rho}{\partial T}\right)_{p}\left[\frac{1}{u^{2}}-\left(\frac{\partial \rho}{\partial p}\right)_{T}\right]^{-1}$

$\left(\frac{\partial \rho}{\partial T}\right)_{s}=\frac{1}{u^{2}}\left(\frac{\partial p}{\partial T}\right)_{s}$

If rule (2) is applied to the isobaric derivative in (14), it is obtained

$\left[\frac{1}{\partial T}\left(\frac{\partial \rho}{\partial T}\right)_{p}\right]_{s}=\left(\frac{\partial^{2} \rho}{\partial T^{2}}\right)_{p}+\frac{\partial^{2} \rho}{\partial T \partial p}\left(\frac{\partial p}{\partial T}\right)_{s}$

The second order isobaric derivative in (16) may be obtained from the following thermodynamic relation [10]

$\left(\frac{\partial^{2} \rho}{\partial T^{2}}\right)_{p}=\frac{\rho^{2}}{T}\left(\frac{\partial c_{p}}{\partial p}\right)_{T}+\frac{2}{\rho}\left(\frac{\partial \rho}{\partial T}\right)_{p}^{2}$

The specific heat capacity at constant pressure may be obtained from

$c_{p}=\frac{T}{\rho^{2}}\left(\frac{\partial \rho}{\partial T}\right)_{p}^{2}\left[\left(\frac{\partial \rho}{\partial p}\right)_{T}-\frac{1}{u^{2}}\right]^{-1}$

and finally

$c_{v}=\frac{c_{p}}{u^{2}}\left(\frac{\partial \rho}{\partial p}\right)_{T}^{-1}$

2.2 Density and entropy as independent variables

2.2.1 Implicit use of entropy

In this case, one can start from the relation [10]

$\left(\begin{array}{l}\partial p \\ \partial \rho\end{array}\right)_{s}=u^{2}$

and the following property relation [10]

$\left(\frac{\partial T}{\partial \rho}\right)_{s}=\frac{1}{\rho^{2}}\left(\frac{\partial p}{\partial s}\right)_{\rho}$

In order to eliminate $s$ at R.H.S. of (21), one can use rule (4) to obtain 
$\left(\frac{\partial p}{\partial s}\right)_{\rho}=\left(\frac{\partial T}{\partial s}\right)_{\rho}\left(\frac{\partial p}{\partial T}\right)_{\rho}$

If (22) is combined with the following relation [10]

$c_{v}=T\left(\frac{\partial s}{\partial T}\right)_{\rho}$

it becomes

$\left(\frac{\partial p}{\partial s}\right)_{\rho}=\frac{T}{c_{v}}\left(\frac{\partial p}{\partial T}\right)_{\rho}$

Combining (21) and (24) one obtains

$\left(\frac{\partial T}{\partial \rho}\right)_{s}=\frac{T}{\rho^{2} c_{v}}\left(\frac{\partial p}{\partial T}\right)_{\rho}$

However, Eqs. (20) and (25) may not be solved for $p$ and $T$ since $c_{v}$ is also unknown. This may be overcome by introducing the following relation [10]

$\left(\frac{\partial c_{v}}{\partial \rho}\right)_{T}=-\frac{T}{\rho^{2}}\left(\frac{\partial^{2} p}{\partial T^{2}}\right)_{\rho}$

Having in mind rule (2) one can write

$\left(\frac{\partial c_{v}}{\partial \rho}\right)_{s}=\left(\frac{\partial c_{v}}{\partial \rho}\right)_{T}+\left(\frac{\partial c_{v}}{\partial T}\right)_{\rho}\left(\frac{\partial T}{\partial \rho}\right)_{s}$

Combining (26) and (27) one obtains

$\left(\frac{\partial c_{v}}{\partial \rho}\right)_{s}=-\frac{T}{\rho^{2}}\left(\frac{\partial^{2} p}{\partial T^{2}}\right)_{\rho}+\left(\frac{\partial c_{v}}{\partial T}\right)_{\rho}\left(\frac{\partial T}{\partial \rho}\right)_{s}$

When $c_{v}$ is known, $c_{p}$ may be calculated from

$c_{p}=c_{v} u^{2}\left[u^{2}-\frac{T}{\rho^{2} c_{v}}\left(\frac{\partial p}{\partial T}\right)_{\rho}^{2}\right]^{-1}$

and the following property relation [10]

$\left(\frac{\partial T}{\partial \rho}\right)_{s}=\frac{1}{\rho^{2}}\left(\frac{\partial p}{\partial s}\right)_{\rho}$

Now, $c_{v}$ is calculated from [10]
$c_{v}=T\left(\frac{\partial T}{\partial s}\right)_{\rho}^{-1}$

According to the rule (2), one can write

$\left(\frac{\partial p}{\partial \rho}\right)_{s}=\left(\frac{\partial p}{\partial \rho}\right)_{T}+\left(\frac{\partial p}{\partial T}\right)_{\rho}\left(\frac{\partial T}{\partial \rho}\right)$

Combining (30), (31), and (33) one obtains

$\left(\frac{\partial p}{\partial \rho}\right)_{T}=u^{2}-\frac{1}{\rho^{2}}\left(\frac{\partial p}{\partial s}\right)_{\rho}\left(\frac{\partial p}{\partial T}\right)$

and finally [10]

$c_{p}=c_{v} u^{2}\left(\frac{\partial p}{\partial \rho}\right)_{T}^{-1}$

2.3 Pressure and entropy as independent variables In this case, one can start from the relation [10]

$\left(\frac{\partial \rho}{\partial p}\right)_{s}=\frac{1}{u^{2}}$

and the following property relation [10]

$\left(\frac{\partial T}{\partial p}\right)_{s}=-\frac{1}{\rho^{2}}\left(\frac{\partial \rho}{\partial s}\right)_{p}$

In order to eliminate $s$ at R.H.S. of (37), one can use rule (4) to obtain

$\left(\frac{\partial \rho}{\partial s}\right)_{p}=\left(\frac{\partial T}{\partial s}\right)_{p}\left(\frac{\partial \rho}{\partial T}\right)_{p}$

If (38) is combined with the following relation [10]

$c_{p}=T\left(\frac{\partial s}{\partial T}\right)_{p}$

(29) it becomes

$\left(\frac{\partial \rho}{\partial s}\right)_{p}=\frac{T}{c_{p}}\left(\frac{\partial \rho}{\partial T}\right)_{p}$

Combining (37) and (40) one obtains

$\left(\frac{\partial T}{\partial p}\right)_{s}=-\frac{T}{\rho^{2} c_{p}}\left(\frac{\partial \rho}{\partial T}\right)_{p}$

However, Eqs. (36) and (41) may not be solved for $\rho$ and $T$ since $c_{p}$ is also unknown. This may be overcome by introducing the following relation [10]

$\left(\frac{\partial c_{p}}{\partial p}\right)_{T}=-\frac{T}{\rho^{2}}\left[\frac{2}{\rho}\left(\frac{\partial \rho}{\partial T}\right)_{p}^{2}-\left(\frac{\partial^{2} \rho}{\partial T^{2}}\right)_{p}\right]$ 
Having in mind rule (2) one can write

$$
\left(\frac{\partial c_{p}}{\partial p}\right)_{s}=\left(\frac{\partial c_{p}}{\partial p}\right)_{T}+\left(\frac{\partial c_{p}}{\partial T}\right)_{p}\left(\frac{\partial T}{\partial p}\right)_{s}
$$

Combining (42) and (43) one obtains

$$
\left(\frac{\partial c_{p}}{\partial p}\right)_{s}=-\frac{T}{\rho^{2}}\left[\frac{2}{\rho}\left(\frac{\partial \rho}{\partial T}\right)_{p}^{2}-\left(\frac{\partial^{2} \rho}{\partial T^{2}}\right)_{p}\right]+\left(\frac{\partial c_{p}}{\partial T}\right)_{p}\left(\frac{\partial T}{\partial p}\right)_{s}
$$

When $c_{p}$ is known, $c_{v}$ may be calculated from

$c_{v}=\frac{c_{p}}{u^{2}}\left[\frac{1}{u^{2}}+\frac{T}{\rho^{2} c_{p}}\left(\frac{\partial \rho}{\partial T}\right)_{p}^{2}\right]^{-1}$

\section{Algorithms of solution}

In this paper it is supposed that practical speed of sound measurements are conducted in $p-T$ domain bounded by two isentropes and two isotherms, isochores, or isobars, respectively, or in any other domain from which speed of sound data can be mapped into the former domains with negligible error. Since true isentropes are yet to be found, approximate ones are generated by a cubic equation of state (EOS). For that purpose the following relation is used

$$
s_{2}-s_{1}=s_{2}^{R}-s_{1}^{R}+\Delta s^{i g}
$$

where entropy change, $s_{2}-s_{1}$, between any two states 1 and 2 is represented as a sum of residual entropy change, $s_{2}{ }^{R}-$ $s_{1}{ }^{R}$, and ideal gas entropy change $\Delta s^{i g}$. The former is obtained from an EOS, while the later is given by

$\Delta s^{i g}=\int_{T_{1}}^{T_{2}} c_{p}^{i g}(T) \frac{d T}{T}-R \ln \left(\frac{p_{2}}{p_{1}}\right)$

where $c_{p}{ }^{i g}$ is the ideal gas heat capacity. Since along an isentrope it holds

$s_{2}-s_{1}=0$

Eqs. (47) and (48) are replaced into (46), and the resulting equation

$s_{2}^{R}-s_{1}^{R}+\int_{T_{1}}^{T_{2}} c_{p}^{i g}(T) \frac{d T}{T}-R \ln \left(\frac{p_{2}}{p_{1}}\right)=0$

is solved for $p_{2}$ or $T_{2}$, given $p_{1}$ and $T_{1}$. Since Eq. (49) is nonlinear with respect to $p_{2}$ and $T_{2}$, the solution is obtained by iteration. If $u$ is specified along isotherms or along isochores, specific volume or temperature is iterated, respectively, and pressure is obtained from an EOS directly, since the cubic EOSs are pressure explicit. However, if $u$ is specified along isobars, temperature is iterated in outer loop (finding zero of Eq. (49)), and specific volume in inner loop (finding zero of an EOS).

\subsection{Temperature and entropy as independent variables}

When it comes to gaseous phase above critical temperature, the most common way of deriving thermodynamic properties from speed of sound is the one based on numerical integration with respect to temperature, along paths of constant density. Although this approach is more demanding (e.g., computationally and experimentally) than the one conducted along paths of constant pressure, it is proved to be more stable. The main source of instability in the later approach are isobaric derivatives, which are estimated less accurately than isochoric ones in the former approach. The aim of the following two algorithms is to check out if isochoric derivatives also introduce less error than isobaric ones when entropy is used instead of density (in the former approach) or pressure (in the latter approach) as independent variable.

\subsubsection{Isothermal and isochoric derivatives}

1. Specify $u(p, T)$ at several isotherms, along several isentropes generated by a cubic EOS

2. Specify $\rho$ and $c_{v}$ or $(\partial p / \partial T)_{\rho}$ at several pressures along isotherm with lowest temperature $\left(T_{0}\right)$

\section{Estimate $(\partial p / \partial \rho)_{T}$}

4. Calculate $c_{v}$ and $c_{p}$ from (10) and (11), respectively

5. Estimate $\partial^{2} p / \partial T \partial \rho$ and $\left(\partial c_{v} / \partial \rho\right)_{T}$

6. Calculate $(\partial \rho / \partial T)_{s}, \quad(\partial p / \partial T)_{s}, \quad\left[\partial(\partial p / \partial T)_{\rho} / \partial T\right]_{s}$, and $\left(\partial^{2} p / \partial T^{2}\right)_{\rho}$ from (6), (7), (8), and (9), respectively

7. Calculate $\rho, p$, and $(\partial p / \partial T) \rho$ at $T=T_{0}+\Delta T$ by numerical integration of $(6),(7)$, and (8), respectively

8. Interpolate EOS pressures along EOS isentropes to $T$

9. Interpolate $u$ along EOS isentropes to $T$

10. Interpolate $u$ from EOS pressures to those from step 7

11. Repeat steps 3 to 10 until final temperature is reached

\subsubsection{Isothermal and isobaric derivatives}

1. Specify $u(p, T)$ at several isotherms, along several isentropes generated by a cubic EOS

2. Specify $p$ and $c_{p}$ or $(\partial \rho / \partial T)_{p}$ at several densities along isotherm with lowest temperature $\left(T_{0}\right)$

\section{Estimate $(\partial \rho / \partial p)_{T}$}

4. Calculate $c_{p}$ and $c_{v}$ from (18) and (19), respectively

5. Estimate $\partial^{2} \rho / \partial T \partial p$ and $\left(\partial c_{p} / \partial p\right)_{T}$

6. Calculate $(\partial p / \partial T)_{s}, \quad(\partial \rho / \partial T)_{s}, \quad\left[\partial(\partial \rho / \partial T)_{p} / \partial T\right]_{s}$, and $\left(\partial^{2} \rho / \partial T^{2}\right)_{p}$ from (14), (15), (16), and (17), respectively

7. Calculate $p, \rho$, and $(\partial \rho / \partial T)_{p}$ at $T=T_{0}+\Delta T$ by numerical integration of (14), (15), and (16), respectively 
8. Interpolate EOS pressures along EOS isentropes to $T$

9. Interpolate $u$ along EOS isentropes to $T$

10. Interpolate $u$ from EOS pressures to those from step 7

11. Repeat steps 3 to 10 until final temperature is reached

\subsection{Density and entropy as independent variables}

Thermodynamic properties of gases above their critical temperature may be derived from speed of sound also if numerical integration is conducted with respect to density, along paths of constant temperature. Since this approach requires boundary values along the lowest temperature(s), the aim of the following two algorithms is to check out if the solution is stable without boundary values imposed when integration is conducted along isentropes instead of isotherms. It would be also interesting to compare algorithm which uses entropy explicitly to the one which uses entropy implicitly, because the former solves first order PDEs, while the later solves second order PDEs. Since both temperature and pressure are dependent variables here, it would be also interesting to see if fitted values of speed of sound could successfully replace interpolated ones.

\subsubsection{Implicit use of entropy}

1. Specify $u(p, T)$ at several isochores and several isentropes generated by a cubic EOS

2. Fit $u$ from step 1 to a suitable function of $p$ and $T$

3. Specify $p$ and $c_{v}$ at several temperatures along isochore with lowest density $\left(\rho_{0}\right)$

4. Estimate $(\partial p / \partial T)_{\rho},\left(\partial^{2} p / \partial T^{2}\right)_{\rho}$, and $\left(\partial c_{v} / \partial T\right)_{\rho}$

5. Calculate $(\partial p / \partial \rho)_{s},(\partial T / \partial \rho)_{s}$, and $\left(\partial c_{v} / \partial \rho\right)_{s}$ from (20), (25), and (28), respectively

6. Calculate $p, T$, and $c_{v}$ at $\rho=\rho_{0}+\Delta \rho$ by numerical integration of (20), (25), and (28), respectively

7. Calculate $c_{p}$ from (29)

8. Calculate $u$ at $p$ and $T$ from step 6 by a function from step 2.

9. Repeat steps 4 to 8 until final density is reached

\subsubsection{Explicit use of entropy}

1. Specify $u(p, T)$ at several isochores and several isentropes generated by a cubic EOS

2. Fit $u$ from step 1 to a suitable function of $p$ and $T$

3. Specify $p$ and $c_{v}$ at several temperatures along isochore with lowest density $\left(\rho_{0}\right)$

4. Calculate $s$ from (23) along $\rho_{0}$, at temperatures from step

3 , with initial value chosen arbitrarily

5. Estimate $(\partial p / \partial s)_{\rho}$ and $(\partial T / \partial s)_{\rho}$
6. Calculate $(\partial p / \partial T)_{\rho}=(\partial p / \partial s)_{\rho} /(\partial T / \partial s)_{\rho}$

7. Calculate $c_{v}$ from (32)

8. Calculate $(\partial p / \partial \rho)_{T}$ from (34)

9. Calculate $c_{p}$ from (35)

10. Calculate $(\partial p / \partial \rho)_{s}$ and $(\partial T / \partial \rho)_{s}$ from (30) and (31), respectively

11. Calculate $p$ and $T$ at $\rho=\rho_{0}+\Delta \rho$ by numerical integration of (30) and (31), respectively

12. Calculate $u$ at $p$ and $T$ from step 11 by a function from step 2

13. Repeat steps 5 to 12 until final density is reached

\subsection{Pressure and entropy as independent variables}

When thermodynamic properties of liquids are derived from speed of sound, pressure is always used as a variable with respect to which the integration is performed, and the other independent variable is usually temperature. Integration domain is situated between melting line at the left and saturation line at the right, and since these two are not isotherms, significant part of the domain is left uncovered. However, it can be increased considerably if integration is performed along isentropes instead of isotherms. When it comes to gaseous phase, integration with respect to pressure is not stable, even if isothermal paths are replaced by isentropic ones. However, it would be interesting to see if isentropic paths combined with approach similar to the one tried before [9] may solve the problem, especially having in mind aggravating circumstance that boundary values may not be specified along isentrope(s).

\subsubsection{Numerical integration}

1. Specify $u(p, T)$ at several isobars, along several isentropes generated by a cubic EOS

2. Specify $\rho$ and $c_{p}$ at several temperatures along isobar with lowest pressure $\left(p_{0}\right)$

3. Estimate $(\partial \rho / \partial T)_{p},\left(\partial^{2} \rho / \partial T^{2}\right)_{p}$, and $\left(\partial c_{p} / \partial T\right)_{p}$

4. Calculate $(\partial \rho / \partial p)_{s},(\partial T / \partial p)_{s}$, and $\left(\partial c_{p} / \partial p\right)_{s}$ from (36), (41), and (44), respectively

5. Calculate $\rho, T$, and $c_{p}$ at $p=p_{0}+\Delta p$ by numerical integration of (36), (41), and (44), respectively

6. Calculate $c_{v}$ from (45)

7. Interpolate $u$ along EOS isentropes to $p$

8. Interpolate $u$ from EOS temperatures to those from step 5

9. Repeat steps 3 to 8 until final pressure is reached 


\subsubsection{Least squares}

1. Specify $u(p, T)$ at several isobars, along several isentropes generated by a cubic EOS

2. Specify $\rho$ and $c_{p}$ at several temperatures along isobar with lowest pressure $\left(p_{0}\right)$

3. Calculate $s$ from (39) along $p_{0}$, at temperatures from step

2 , with initial value chosen arbitrarily

4. Guess $\rho$ along isentropes from step 3 at several pressures

5. Estimate $(\partial \rho / \partial p)_{s}$

6. Calculate $u$ from (36)

7. Interpolate $T$ with respect to $u$ and $p$

8. Estimate $(\partial T / \partial p)_{s},(\partial \rho / \partial s)_{p}$, and $(\partial T / \partial s)_{p}$

9. Calculate $(\partial \rho / \partial T)_{p}=(\partial \rho / \partial s)_{p} /(\partial T / \partial s)_{p}$

10. Calculate $c_{p}$ from (39)

11. Calculate $c_{v}$ from (45)

12. Calculate $f=(\partial T / \partial p)_{s}+(\partial \rho / \partial s)_{p} / \rho^{2}$

13. Calculate $g=\left(\Sigma f^{2}\right) / 2$

14. If $g>10^{-4}$, calculate new values of $\rho$ by a least squares method

15 . Repeat steps 5 to 14 as many times as necessary to obtain $g \leq 10^{-4}$

\section{Results and discussion}

All interpolations are performed by polynomials [11], while derivatives are estimated by cubic splines [12] and interpolation polynomials. When both pressure and temperature are dependent variables (Algorithms 3.2.1 and 3.2.2), speed of sound data are fitted to bivariate Chebyshev polynomials (with $\ln (p)$ and $\ln (T)$ scaled between -1 and +1 ). The polynomials coefficients are obtained by method of linear least squares with iterative refinement of Björck [13]. Numerical integrations are performed by Runge-KuttaVerner fifth-order and sixth-order method with adaptive stepsize [14]. In Algorithm 3.3.2, densities are calculated by a modified Levenberg - Marquardt method [15 - 17]. Algorithms of solution described in Sections 3.1 and 3.2 (for gaseous phase) and 3.3 (for liquid and gaseous phase) are tested with several substances. Their list and $p-\rho-T$ ranges covered are given in Tables 1 to 4, 16, 19, and 20. Reference properties are generated by corresponding fundamental EOSs [18 - 24]. Speed of sound data used are obtained from the same EOSs and from measurements [3]. All the results obtained as well as the coefficients of Chebyshev polynomials (see appendix) are given in separate file as a supplement to this paper.

Initial values for supercritical gaseous phase (Algorithms 3.1.1 and 3.1.2) are specified along isotherm with the lowest temperature, at 10 equally spaced pressures (see Table 5). Reference properties are specified at 16 equally spaced isotherms, along 10 isentropes passing through the points with initial values. Speed of sound data are specified at the same isotherms, but along isentropes generated by PengRobinson (P-R) EOS [25]. All derivatives are estimated by cubic splines. Both thermal and caloric properties are derived with AADs an order of magnitude smaller if isochoric derivatives are used instead of isobaric ones (see Tables 9 and 10). These AADs are of similar magnitude to those when temperature and density [4] or temperature and pressure [1], respectively, are used as independent variables.

Initial values for supercritical gaseous phase (Algorithms 3.2.1 and 3.2.2) are specified along isochore with the lowest density, at 11 equally spaced temperatures (see Table 6). Entropies corresponding to these temperatures are obtained from Eq. (23), with initial values chosen arbitrarily. Reference properties are specified at 11 isochores, along 11 isentropes passing through the points with initial values. Speed of sound data are specified at isochores and isentropes generated by P-R EOS [25]. Derivatives are estimated by interpolation polynomials (Algorithm 3.2.1) and cubic splines (Algorithm 3.2.2). Both thermal and caloric properties are derived with AADs about 50\% smaller if entropy is used explicitly instead of implicitly (see Tables 11 and 12). The solution is stable without boundary values imposed when integration is conducted along isentropes instead of isotherms. Fitted values of speed of sound can successfully replace interpolated ones.

Initial values for transcritical gaseous phase (Algorithms 3.1.1 and 3.1.2) are specified along isotherm with the lowest temperature, at 10 equally spaced pressures (see Table 7). Reference properties are specified at 11 to 15 equally spaced isotherms, along 10 isentropes passing through the points with initial values. Speed of sound data are specified at the same isotherms, but along isentropes generated by P-R EOS [25]. All derivatives are estimated by cubic splines. Both thermal and caloric properties are derived with AADs 4 to 5 times smaller if isochoric derivatives are used instead of isobaric ones (see Tables 13 and 14). These AADs are of similar magnitude to those when temperature and ratio of density to saturated vapor density [5], or temperature and ratio of pressure to saturation pressure [2], respectively, are used as independent variables.

Initial values for liquid phase (Algorithm 3.3.1) are specified along isobar with the lowest pressure, at 7 to 10 equally spaced temperatures (see Table 8). Reference properties are specified at 9 to 11 isobars, along 7 to 10 isentropes passing through the points with initial values. Speed of sound data are specified at the same isobars, but along isentropes generated by P-R EOS [25]. All derivatives are estimated by interpolation polynomials. Both thermal and caloric properties are derived with AADs of similar magnitude to those when pressure and temperature $[6,7]$ are used as independent variables (see Table 15). It is confirmed that stability of solution in liquid phase, when integration is performed along isentropes, is similar to that along isotherms [26].

Initial values for supercritical gaseous phase (Algorithm 3.3.2) are specified along isobar with the lowest pressure, at 11 equally spaced temperatures (see Table 17). Entropies corresponding to these temperatures are obtained from Eq. (39), with initial values chosen arbitrarily. Reference properties are specified at 11 isobars, along 11 isentropes passing through the points with initial values. Speed of sound data are specified at the same isobars, but along isentropes generated by P-R EOS [25]. All derivatives are estimated by 
interpolation polynomials. The results are the same whether guessed values of density are generated by reference EOS (with simulated error of $5 \%$ ) or by P-R EOS. Both thermal and caloric properties are derived with AADs of similar magnitude to those when density and temperature $[4,9]$ are used as independent variables (see Table 18). The solution is obtained even without boundary values imposed, but with initial values consisting of thermal and caloric properties.

Initial values for argon in supercritical gaseous phase (Algorithm 3.1.1) are specified along isotherm with the lowest temperature, at 11 equally spaced pressures (see Table 21). Reference properties are specified at 10 isotherms, along 11 isentropes passing through the points with initial values. Speed of sound data $[3,18]$ are specified at the same isotherms, but along isentropes generated by P-R EOS [25]. All derivatives are estimated by cubic splines. Similar results are obtained with measured and EOS generated speed of sound values, when these later are specified in the same ( $p$, $T$ ) points in which speed of sound measurements are conducted (see Table 23).

Initial values for carbon dioxide in transcritical gaseous phase (Algorithm 3.1.1) are specified along isotherm with the lowest temperature, at 8 equally spaced pressures (see Table 22). Reference properties are specified at 9 isotherms, along 9 isentropes passing through the points with initial values. Speed of sound data [3, 23] are specified at the same isotherms, but along isentropes generated by P-R EOS [25]. All derivatives are estimated by cubic splines. Similar results are obtained with measured and EOS generated speed of sound values, when these later are specified in the same ( $p$, $T$ ) points in which speed of sound measurements are conducted (see Table 24).

The fact that similar results are obtained with measured and EOS generated speed of sound values, in the last two cases, is confirmation that the two sets of speed of sound have similar uncertainty. So, why AADs in Tables 9 and 23 for argon, or 13 and 24 for carbon dioxide, are an order of magnitude different? This question may be best answered if one takes a closer look at corresponding data sets for argon and carbon dioxide. From Table 1 one can see that temperature range covered for argon is 160 to $310 \mathrm{~K}$ and the number of isotherms used is 16 , while in Table 20 the corresponding range is 156.08 to $350 \mathrm{~K}$ and the number of experimental isotherms is only 10 . Also, from Table 3 one can see that temperature range covered for carbon dioxide is 240 to $480 \mathrm{~K}$ and the number of isotherms used is 13 , while in Table 20 the corresponding range is 250 to $450 \mathrm{~K}$ and the number of experimental isotherms is only 6 .

\section{Conclusions}

With only a few initial data, specified at single isotherm, isochore, or isobar, it is possible to derive thermal and caloric properties of real fluids from speed of sound in a wide $p-T$ range if integration paths follow isentropes. While isentropic paths enable covering wider pressure range in gaseous phase, comparing to isochoric paths, this advantage comes at a price. Namely, quicker rise of pressure with temperature along isentropes results in higher nonlinearities, which introduces additional error during interpolation and derivation. It is especially the case when integration is conducted with respect to temperature, but somewhat less pronounced when integration is conducted with respect to density. Besides, selection of appropriate initial temperature and initial pressure range, or initial density and initial temperature range, is of crucial significance if one wants to cover specific area of thermodynamic surface in gaseous phase. Since isentropes of liquids have similar shape to isotherms in $p-T$ coordinates, the solution stability is similar too. However, their positive inclination with respect to isotherms enables wider temperature range to be covered. Generally, the AADs of the results obtained, with respect to corresponding reference data, are such that their uncertainties are similar to those of direct measurements.

\section{Nomenclature}

AAD average absolute deviation (\%)

$c_{p} \quad$ specific heat capacity at constant pressure $(\mathrm{J} / \mathrm{kgK})$

$c_{v} \quad$ specific heat capacity at constant volume $(\mathrm{J} / \mathrm{kgK})$

$p \quad$ pressure $(\mathrm{Pa})$

$s \quad$ specific entropy $(\mathrm{J} / \mathrm{kgK})$

$T \quad$ thermodynamic temperature $(\mathrm{K})$

$u \quad$ speed of sound $(\mathrm{m} / \mathrm{s})$

$v \quad$ specific volume $\left(\mathrm{m}^{3} / \mathrm{kg}\right)$

\section{Greek Letters}

$\rho \quad$ mass density $\left(\mathrm{kg} / \mathrm{m}^{3}\right)$

\section{Superscripts}

ig ideal gas

$R \quad$ residual

Abbreviations

EOS equation of state

PDE partial differential equation

P-R Peng-Robinson

\section{Appendix}

Table 1. $p-\rho-T$ ranges covered in supercritical gaseous phase (Algorithms 3.1.1 and 3.1.2).

\begin{tabular}{|l|c|c|c|c|c|c|}
\hline \multirow{2}{*}{} & \multicolumn{2}{|c|}{$p(\mathrm{MPa})$} & \multicolumn{2}{c|}{$\rho\left(\mathrm{kg} \cdot \mathrm{m}^{-3}\right)$} & \multicolumn{2}{c|}{$T(\mathrm{~K})$} \\
\cline { 2 - 7 } & Min & Max & Min & Max & Min & Max \\
\hline $\mathrm{Ar}$ & 0.50 & 29.5246 & 15.4656 & 455.7321 & 160.0 & 310.0 \\
\hline $\mathrm{N}_{2}$ & 0.35 & 41.5905 & 8.6420 & 381.1771 & 140.0 & 290.0 \\
\hline $\mathrm{O}_{2}$ & 0.50 & 41.2324 & 11.6120 & 457.2604 & 170.0 & 320.0 \\
\hline $\mathrm{CH}_{4}$ & 0.50 & 49.2063 & 4.9837 & 231.6339 & 200.0 & 350.0 \\
\hline $\mathrm{CO}_{2}$ & 0.70 & 35.1821 & 11.9116 & 453.9972 & 320.0 & 470.0 \\
\hline $\mathrm{H}_{2} \mathrm{O}$ & 2.20 & 51.8782 & 7.4587 & 218.9286 & 660.0 & 810.0 \\
\hline
\end{tabular}

Table 2. $p-\rho-T$ ranges covered in supercritical gaseous phase (Algorithms 3.2.1 and 3.2.2).

\begin{tabular}{|l|c|c|c|c|c|c|}
\hline \multirow{2}{*}{} & \multicolumn{2}{|c|}{$p(\mathrm{MPa})$} & \multicolumn{2}{c|}{$\rho\left(\mathrm{kg} \cdot \mathrm{m}^{-3}\right)$} & \multicolumn{2}{c|}{$T(\mathrm{~K})$} \\
\cline { 2 - 7 } & Min & Max & Min & Max & Min & Max \\
\hline $\mathrm{Ar}$ & 5.0 & 63.5603 & 243.2643 & 500.0 & 160.0 & 489.4233 \\
\hline $\mathrm{N}_{2}$ & 3.5 & 76.0620 & 120.9870 & 375.0 & 140.0 & 455.4120 \\
\hline $\mathrm{O}_{2}$ & 5.0 & 73.7194 & 162.6782 & 460.0 & 170.0 & 470.1185 \\
\hline $\mathrm{CH}_{4}$ & 5.0 & 85.4198 & 87.76400 & 240.0 & 200.0 & 463.6393 \\
\hline $\mathrm{CO}_{2}$ & 7.0 & 72.2296 & 178.7401 & 530.0 & 320.0 & 608.2809 \\
\hline $\mathrm{H}_{2} \mathrm{O}$ & 22.0 & 98.9995 & 141.9413 & 290.0 & 660.0 & 957.4060 \\
\hline
\end{tabular}


Table 3. $p-\rho-T$ ranges covered in transcritical gaseous phase (Algorithms 3.1.1 and 3.1.2).

\begin{tabular}{|l|c|c|c|c|c|c|}
\hline \multirow{2}{*}{} & \multicolumn{2}{|c|}{$p(\mathrm{MPa})$} & \multicolumn{2}{c|}{$\rho\left(\mathrm{kg} \cdot \mathrm{m}^{-3}\right)$} & \multicolumn{2}{c|}{$T(\mathrm{~K})$} \\
\cline { 2 - 7 } & Min & Max & Min & Max & Min & Max \\
\hline $\mathrm{Ar}$ & 0.1 & 21.7206 & 4.0577 & 252.3890 & 120.0 & 400.0 \\
\hline $\mathrm{N}_{2}$ & 0.1 & 33.2891 & 3.1089 & 306.9767 & 110.0 & 310.0 \\
\hline $\mathrm{O}_{2}$ & 0.1 & 36.8089 & 3.1174 & 358.3584 & 125.0 & 365.0 \\
\hline $\mathrm{CH}_{4}$ & 0.1 & 48.0311 & 1.2617 & 190.7753 & 155.0 & 415.0 \\
\hline $\mathrm{CO}_{2}$ & 0.1 & 19.1436 & 2.2282 & 240.7653 & 240.0 & 480.0 \\
\hline $\mathrm{H}_{2} \mathrm{O}$ & 0.1 & 6.9887 & 0.4738 & 22.7240 & 460.0 & 720.0 \\
\hline
\end{tabular}

Table 4. $p-\rho-T$ ranges covered in liquid phase (Algorithm 3.3.1).

\begin{tabular}{|l|c|c|c|c|c|c|}
\hline \multirow{2}{*}{} & \multicolumn{2}{|c|}{$p(\mathrm{MPa})$} & \multicolumn{2}{c|}{$\rho\left(\mathrm{kg} \cdot \mathrm{m}^{-3}\right)$} & \multicolumn{2}{c|}{$T(\mathrm{~K})$} \\
\cline { 2 - 7 } & Min & Max & Min & Max & Min & Max \\
\hline $\mathrm{Ar}$ & 5.0 & 100.0 & 1053.233 & 1495.712 & 90.0 & 187.7662 \\
\hline $\mathrm{N}_{2}$ & 3.5 & 90.0 & 604.0069 & 917.1604 & 70.0 & 158.0081 \\
\hline $\mathrm{O}_{2}$ & 5.0 & 80.0 & 845.5365 & 1338.650 & 60.0 & 176.0196 \\
\hline $\mathrm{CH}_{4}$ & 5.0 & 80.0 & 323.9083 & 469.7829 & 100.0 & 209.8933 \\
\hline $\mathrm{CO}_{2}$ & 7.0 & 100.0 & 914.2519 & 1253.424 & 220.0 & 328.1373 \\
\hline $\mathrm{H}_{2} \mathrm{O}$ & 0.1 & 900.0 & 967.4033 & 1220.401 & 280.0 & 402.2188 \\
\hline
\end{tabular}

Table 5. Points with initial values in supercritical gaseous phase (Algorithms 3.1.1 and 3.1.2).

\begin{tabular}{|l|c|c|c|c|}
\hline \multirow{2}{*}{} & \multicolumn{3}{|c|}{$p(\mathrm{MPa})$} & \multirow{2}{*}{$T(\mathrm{~K})$} \\
\cline { 2 - 4 } & Min & Max & Step & \\
\hline $\mathrm{Ar}$ & 0.50 & 5.0 & 0.50 & 160.0 \\
\hline $\mathrm{N}_{2}$ & 0.35 & 3.5 & 0.35 & 140.0 \\
\hline $\mathrm{O}_{2}$ & 0.50 & 5.0 & 0.50 & 170.0 \\
\hline $\mathrm{CH}_{4}$ & 0.50 & 5.0 & 0.50 & 200.0 \\
\hline $\mathrm{CO}_{2}$ & 0.70 & 7.0 & 0.70 & 320.0 \\
\hline $\mathrm{H}_{2} \mathrm{O}$ & 2.20 & 22.0 & 2.20 & 660.0 \\
\hline
\end{tabular}

Table 6. Points with initial values in supercritical gaseous phase (Algorithms 3.2.1 and 3.2.2).

\begin{tabular}{|l|c|c|c|c|}
\hline \multirow{2}{*}{$\mathrm{Ar}$} & \multicolumn{3}{|c|}{$T(\mathrm{~K})$} & \multirow{2}{*}{$\rho\left(\mathrm{kg} \cdot \mathrm{m}^{-3}\right)$} \\
\cline { 2 - 5 } & Min & Max & Step & \\
\hline $\mathrm{N}_{2}$ & 160.0 & 260.0 & 10.0 & 243.2643 \\
\hline $\mathrm{O}_{2}$ & 170.0 & 240.0 & 10.0 & 120.9870 \\
\hline $\mathrm{CH}_{4}$ & 200.0 & 300.0 & 10.0 & 87.76400 \\
\hline $\mathrm{CO}_{2}$ & 320.0 & 420.0 & 10.0 & 178.7401 \\
\hline $\mathrm{H}_{2} \mathrm{O}$ & 660.0 & 760.0 & 10.0 & 141.9413 \\
\hline
\end{tabular}

Table 7. Points with initial values in transcritical gaseous phase (Algorithms 3.1.1 and 3.1.2).

\begin{tabular}{|l|c|c|c|c|}
\hline \multirow{2}{*}{} & \multicolumn{3}{|c|}{$p(\mathrm{MPa})$} & \multirow{2}{*}{$T(\mathrm{~K})$} \\
\cline { 2 - 5 } & Min & Max & Step & \\
\hline $\mathrm{N}_{2}$ & 0.1 & 1.0 & 0.1 & 120.0 \\
\hline $\mathrm{O}_{2}$ & 0.1 & 1.0 & 0.1 & 110.0 \\
\hline $\mathrm{CH}_{4}$ & 0.1 & 1.0 & 0.1 & 125.0 \\
\hline $\mathrm{CO}_{2}$ & 0.1 & 1.0 & 0.1 & 240.0 \\
\hline $\mathrm{H}_{2} \mathrm{O}$ & 0.1 & 1.0 & 0.1 & 460.0 \\
\hline
\end{tabular}

Table 8. Points with initial values in liquid phase (Algorithm 3.3.1).

\begin{tabular}{|l|c|c|c|c|}
\hline & \multicolumn{3}{|c|}{$T(\mathrm{~K})$} & \multirow{2}{*}{} \\
\cline { 2 - 5 } & Min & Max & Step & $p(\mathrm{MPa})$ \\
\hline $\mathrm{Ar}$ & 90.0 & 135.0 & 5.0 & 5.0 \\
\hline $\mathrm{N}_{2}$ & 70.0 & 115.0 & 5.0 & 3.5 \\
\hline $\mathrm{O}_{2}$ & 60.0 & 140.0 & 10.0 & 5.0 \\
\hline $\mathrm{CH}_{4}$ & 100.0 & 170.0 & 10.0 & 5.0 \\
\hline $\mathrm{CO}_{2}$ & 220.0 & 280.0 & 10.0 & 7.0 \\
\hline $\mathrm{H}_{2} \mathrm{O}$ & 280.0 & 360.0 & 10.0 & 0.1 \\
\hline
\end{tabular}

Table 9. Average absolute deviation in supercritical gaseous phase (Algorithm 3.1.1).

\begin{tabular}{|l|c|c|c|c|}
\hline \multirow{2}{*}{} & \multicolumn{4}{|c|}{ AAD (\%) } \\
\cline { 2 - 5 } & $\rho$ & $p$ & $c_{p}$ & $c_{v}$ \\
\hline $\mathrm{Ar}$ & 0.0006 & 0.0011 & 0.0080 & 0.0061 \\
\hline $\mathrm{N}_{2}$ & 0.0094 & 0.0131 & 0.0709 & 0.0575 \\
\hline $\mathrm{O}_{2}$ & 0.0051 & 0.0075 & 0.0516 & 0.0374 \\
\hline $\mathrm{CH}_{4}$ & 0.0057 & 0.0112 & 0.0961 & 0.0671 \\
\hline $\mathrm{CO}_{2}$ & 0.0031 & 0.0041 & 0.0270 & 0.0177 \\
\hline $\mathrm{H}_{2} \mathrm{O}$ & 0.0059 & 0.0068 & 0.0629 & 0.0340 \\
\hline
\end{tabular}

Table 10. Average absolute deviation in supercritical gaseous phase (Algorithm 3.1.2).

\begin{tabular}{|l|c|c|c|c|}
\hline \multirow{2}{*}{} & \multicolumn{4}{|c|}{ AAD (\%) } \\
\cline { 2 - 5 } & $\rho$ & $p$ & $c_{p}$ & $c_{v}$ \\
\hline $\mathrm{Ar}$ & 0.0230 & 0.0431 & 0.2729 & 0.1857 \\
\hline $\mathrm{N}_{2}$ & 0.0253 & 0.0383 & 0.3050 & 0.2174 \\
\hline $\mathrm{O}_{2}$ & 0.0239 & 0.0352 & 0.2440 & 0.1682 \\
\hline $\mathrm{CH}_{4}$ & 0.1194 & 0.1865 & 1.3816 & 1.0070 \\
\hline $\mathrm{CO}_{2}$ & 0.0275 & 0.0371 & 0.2824 & 0.2234 \\
\hline $\mathrm{H}_{2} \mathrm{O}$ & 0.0320 & 0.0399 & 0.4339 & 0.3562 \\
\hline
\end{tabular}


Table 11. Average absolute deviation in supercritical gaseous phase (Algorithm 3.2.1).

\begin{tabular}{|l|c|c|c|c|}
\hline \multirow{2}{*}{} & \multicolumn{4}{|c|}{ AAD (\%) } \\
\cline { 2 - 5 } & $p$ & $T$ & $c_{p}$ & $c_{v}$ \\
\hline $\mathrm{Ar}$ & 0.0010 & 0.0033 & 0.2979 & 0.1558 \\
\hline $\mathrm{N}_{2}$ & 0.0004 & 0.0014 & 0.0798 & 0.0524 \\
\hline $\mathrm{O}_{2}$ & 0.0001 & 0.0004 & 0.0288 & 0.0189 \\
\hline $\mathrm{CH}_{4}$ & 0.0011 & 0.0031 & 0.1798 & 0.0923 \\
\hline $\mathrm{CO}_{2}$ & 0.0002 & 0.0004 & 0.0438 & 0.0299 \\
\hline $\mathrm{H}_{2} \mathrm{O}$ & 0.0004 & 0.0010 & 0.2117 & 0.1118 \\
\hline
\end{tabular}

Table 12. Average absolute deviation in supercritical gaseous phase (Algorithm 3.2.2).

\begin{tabular}{|l|c|c|c|c|}
\hline \multirow{2}{*}{} & \multicolumn{4}{|c|}{ AAD (\%) } \\
\cline { 2 - 5 } & $p$ & $T$ & $c_{p}$ & $c_{v}$ \\
\hline $\mathrm{Ar}$ & 0.0004 & 0.0010 & 0.0490 & 0.0246 \\
\hline $\mathrm{N}_{2}$ & 0.0003 & 0.0012 & 0.0658 & 0.0457 \\
\hline $\mathrm{O}_{2}$ & 0.0002 & 0.0011 & 0.0616 & 0.0426 \\
\hline $\mathrm{CH}_{4}$ & 0.0006 & 0.0011 & 0.0780 & 0.0467 \\
\hline $\mathrm{CO}_{2}$ & 0.0003 & 0.0007 & 0.0666 & 0.0463 \\
\hline $\mathrm{H}_{2} \mathrm{O}$ & 0.0005 & 0.0011 & 0.2469 & 0.1302 \\
\hline
\end{tabular}

Table 13. Average absolute deviation in transcritical gaseous phase (Algorithm 3.1.1).

\begin{tabular}{|l|c|c|c|c|}
\hline \multirow{2}{*}{} & \multicolumn{4}{|c|}{ AAD (\%) } \\
\cline { 2 - 5 } & $\rho$ & $p$ & $c_{p}$ & $c_{v}$ \\
\hline $\mathrm{Ar}$ & 0.0071 & 0.0116 & 0.0960 & 0.0663 \\
\hline $\mathrm{N}_{2}$ & 0.0031 & 0.0050 & 0.0480 & 0.0306 \\
\hline $\mathrm{O}_{2}$ & 0.0138 & 0.0191 & 0.1352 & 0.1030 \\
\hline $\mathrm{CH}_{4}$ & 0.0213 & 0.0285 & 0.1243 & 0.0995 \\
\hline $\mathrm{CO}_{2}$ & 0.0083 & 0.0101 & 0.0739 & 0.0596 \\
\hline $\mathrm{H}_{2} \mathrm{O}$ & 0.0016 & 0.0018 & 0.0227 & 0.0212 \\
\hline
\end{tabular}

Table 14. Average absolute deviation in transcritical gaseous phase (Algorithm 3.1.2).

\begin{tabular}{|l|c|c|c|c|}
\hline \multirow{2}{*}{} & \multicolumn{4}{|c|}{ AAD (\%) } \\
\cline { 2 - 5 } & $\rho$ & $p$ & $c_{p}$ & $c_{v}$ \\
\hline $\mathrm{Ar}$ & 0.0119 & 0.0178 & 0.1032 & 0.0767 \\
\hline $\mathrm{N}_{2}$ & 0.0440 & 0.0618 & 0.3475 & 0.2813 \\
\hline $\mathrm{O}_{2}$ & 0.0331 & 0.0456 & 0.2994 & 0.2440 \\
\hline $\mathrm{CH}_{4}$ & 0.1575 & 0.2003 & 1.0555 & 0.9065 \\
\hline $\mathrm{CO}_{2}$ & 0.0149 & 0.0175 & 0.1382 & 0.1157 \\
\hline $\mathrm{H}_{2} \mathrm{O}$ & 0.0014 & 0.0016 & 0.0128 & 0.0108 \\
\hline
\end{tabular}

Table 15. Average absolute deviation in liquid phase (Algorithm 3.3.1).

\begin{tabular}{|l|c|c|c|c|}
\hline \multirow{2}{*}{} & \multicolumn{4}{|c|}{ AAD (\%) } \\
\cline { 2 - 5 } & $\rho$ & $T$ & $c_{p}$ & $c_{v}$ \\
\hline $\mathrm{Ar}$ & 0.0001 & 0.0008 & 0.0603 & 0.0256 \\
\hline $\mathrm{N}_{2}$ & 0.0001 & 0.0011 & 0.0839 & 0.0418 \\
\hline $\mathrm{O}_{2}$ & 0.0010 & 0.0023 & 0.0759 & 0.0479 \\
\hline $\mathrm{CH}_{4}$ & 0.0002 & 0.0011 & 0.0908 & 0.0507 \\
\hline $\mathrm{CO}_{2}$ & 0.0003 & 0.0019 & 0.2212 & 0.1032 \\
\hline $\mathrm{H}_{2} \mathrm{O}$ & 0.0004 & 0.0013 & 0.0229 & 0.0186 \\
\hline
\end{tabular}

Table 16. $p-\rho-T$ ranges covered in supercritical gaseous phase (Algorithm 3.3.2).

\begin{tabular}{|l|c|c|c|c|c|c|}
\hline \multirow{2}{*}{} & \multicolumn{2}{|c|}{$p(\mathrm{MPa})$} & \multicolumn{2}{c|}{$\rho\left(\mathrm{kg} \cdot \mathrm{m}^{-3}\right)$} & \multicolumn{2}{c|}{$T(\mathrm{~K})$} \\
\cline { 2 - 7 } & Min & Max & Min & Max & Min & Max \\
\hline $\mathrm{Ar}$ & 5.0 & 15.0 & 63.37348 & 293.2735 & 180.0 & 589.5826 \\
\hline $\mathrm{N}_{2}$ & 3.5 & 14.0 & 32.52045 & 204.8415 & 160.0 & 535.9944 \\
\hline $\mathrm{O}_{2}$ & 5.0 & 15.0 & 49.37306 & 247.1161 & 190.0 & 528.8307 \\
\hline $\mathrm{CH}_{4}$ & 5.0 & 15.0 & 23.28734 & 122.3224 & 220.0 & 521.7476 \\
\hline $\mathrm{CO}_{2}$ & 7.0 & 17.0 & 70.81165 & 278.1599 & 340.0 & 640.0213 \\
\hline $\mathrm{H}_{2} \mathrm{O}$ & 22.0 & 32.0 & 60.44128 & 151.0565 & 680.0 & 956.5035 \\
\hline
\end{tabular}

Table 17. Points with initial values in supercritical gaseous phase (Algorithm 3.3.2).

\begin{tabular}{|l|c|c|c|c|}
\hline \multirow{2}{*}{} & \multicolumn{3}{|c|}{$T(\mathrm{~K})$} & \multirow{2}{*}{} \\
\cline { 2 - 5 } & Min & Max & Step & $p(\mathrm{MPa})$ \\
\hline $\mathrm{Ar}$ & 180.0 & 380.0 & 20.0 & 5.0 \\
\hline $\mathrm{N}_{2}$ & 160.0 & 360.0 & 20.0 & 3.5 \\
\hline $\mathrm{O}_{2}$ & 190.0 & 390.0 & 20.0 & 5.0 \\
\hline $\mathrm{CH}_{4}$ & 220.0 & 420.0 & 20.0 & 5.0 \\
\hline $\mathrm{CO}_{2}$ & 340.0 & 540.0 & 20.0 & 7.0 \\
\hline $\mathrm{H}_{2} \mathrm{O}$ & 680.0 & 880.0 & 20.0 & 22.0 \\
\hline
\end{tabular}

Table 18. AAD and number of iterations taken in supercritical gaseous phase (Algorithm 3.3.2).

\begin{tabular}{|l|c|c|c|c|c|}
\hline \multirow{2}{*}{} & \multirow{2}{*}{ Iterations taken } & \multicolumn{4}{|c|}{ AAD (\%) } \\
\cline { 3 - 6 } & & $\rho$ & $T$ & $c_{p}$ & $c_{v}$ \\
\hline $\mathrm{Ar}$ & 445 & 0.0007 & 0.0033 & 0.1202 & 0.0847 \\
\hline $\mathrm{N}_{2}$ & 334 & 0.0026 & 0.0098 & 0.2915 & 0.2311 \\
\hline $\mathrm{O}_{2}$ & 445 & 0.0011 & 0.0032 & 0.1399 & 0.1089 \\
\hline $\mathrm{CH}_{4}$ & 445 & 0.0010 & 0.0015 & 0.0466 & 0.0350 \\
\hline $\mathrm{CO}_{2}$ & 445 & 0.0005 & 0.0018 & 0.1001 & 0.0695 \\
\hline $\mathrm{H}_{2} \mathrm{O}$ & 556 & 0.0120 & 0.0133 & 0.9247 & 0.2586 \\
\hline
\end{tabular}


Table 19. $p-\rho-T$ ranges covered in supercritical gaseous phase (Algorithm 3.1.1).

\begin{tabular}{|c|c|c|c|c|c|c|}
\hline \multirow{4}{*}{} & \multicolumn{2}{|c|}{$p(\mathrm{MPa})$} & \multicolumn{2}{c|}{$\rho\left(\mathrm{kg} \cdot \mathrm{m}^{-3}\right)$} & \multicolumn{2}{c|}{$T(\mathrm{~K})$} \\
\cline { 2 - 7 } & Min & Max & Min & Max & Min & Max \\
\hline Ar & 0.1 & 16.5101 & 3.0975 & 227.9245 & 156.08 & 350.0 \\
\hline
\end{tabular}

Table 20. $p-\rho-T$ ranges covered in transcritical gaseous phase (Algorithm 3.1.1).

\begin{tabular}{|c|c|c|c|c|c|c|}
\hline \multirow{4}{*}{} & \multicolumn{2}{|c|}{$p(\mathrm{MPa})$} & \multicolumn{2}{c|}{$\rho\left(\mathrm{kg} \cdot \mathrm{m}^{-3}\right)$} & \multicolumn{2}{c|}{$T(\mathrm{~K})$} \\
\cline { 2 - 7 } & Min & Max & Min & Max & Min & Max \\
\hline $\mathrm{CO}_{2}$ & 0.5 & 14.4634 & 11.0975 & 198.7124 & 250.0 & 450.0 \\
\hline
\end{tabular}

Table 21. Points with initial values in supercritical gaseous phase (Algorithms 3.1.1).

\begin{tabular}{|c|c|c|c|c|}
\hline \multirow{2}{*}{} & \multicolumn{3}{|c|}{$p(\mathrm{MPa})$} & \multirow{2}{*}{$T(\mathrm{~K})$} \\
\cline { 2 - 4 } & Min & Max & Step & \\
\hline Ar & 0.1 & 2.1 & 0.2 & 156.08 \\
\hline
\end{tabular}

Table 22. Points with initial values in transcritical gaseous phase (Algorithms 3.1.1).

\begin{tabular}{|c|c|c|c|c|}
\hline \multirow{2}{*}{} & \multicolumn{3}{|c|}{$p(\mathrm{MPa})$} & \multirow{2}{*}{$T(\mathrm{~K})$} \\
\cline { 2 - 5 } & Min & Max & Step & \\
\hline $\mathrm{CO}_{2}$ & 0.5 & 1.2 & 0.1 & 250.0 \\
\hline
\end{tabular}

Table 23. Average absolute deviation in supercritical gaseous phase (Algorithm 3.1.1).

\begin{tabular}{|c|c|c|c|c|}
\hline \multirow{3}{*}{} & \multicolumn{4}{|c|}{ AAD (\%) using measured speed of sound } \\
\cline { 2 - 5 } & $\rho$ & $p$ & $c_{p}$ & $c_{v}$ \\
\hline $\mathrm{Ar}$ & 0.0325 & 0.0356 & 0.1198 & 0.1760 \\
\hline & \multicolumn{5}{|c|}{$\mathrm{AAD}(\%)$ using EOS generated speed of sound } \\
\hline $\mathrm{Ar}$ & 0.0319 & 0.0351 & 0.1202 & 0.1761 \\
\hline
\end{tabular}

Table 24. Average absolute deviation in transcritical gaseous phase (Algorithm 3.1.1).

\begin{tabular}{|c|c|c|c|c|}
\hline \multirow{2}{*}{} & \multicolumn{4}{|c|}{ AAD (\%) using measured speed of sound } \\
\cline { 2 - 5 } & $\rho$ & $p$ & $c_{p}$ & $c_{v}$ \\
\hline $\mathrm{CO}_{2}$ & 0.0845 & 0.0932 & 0.5893 & 0.5254 \\
\hline & \multicolumn{5}{|c|}{ AAD (\%) using EOS generated speed of sound } \\
\hline $\mathrm{CO}_{2}$ & 0.0340 & 0.0403 & 0.3323 & 0.2874 \\
\hline
\end{tabular}

\section{Chebyshev polynomials in two variables}

$$
\begin{aligned}
& T=\ln \left(T_{i, j}\right) \\
& P=\ln \left(P_{i, j}\right) \\
& X_{i, j}=\ln \left(T_{i, j}^{P R}\right) \\
& Y_{i, j}=\ln \left(P_{i, j}^{P R}\right)
\end{aligned}
$$

$X=\frac{2 T-X_{1,1}-X_{N, N}}{X_{N, N}-X_{1,1}}$

$Y=\frac{2 P-Y_{1,1}-Y_{N, N}}{Y_{N, N}-Y_{1,1}}$

$X_{0}=1$

$X_{1}=X$

$X_{2}=2 X^{2}-1$

$X_{3}=4 X^{3}-3 X$

$X_{4}=8 X^{4}-8 X^{2}+1$

$X_{5}=16 X^{5}-20 X^{3}+5 X$

$X_{6}=32 X^{6}-48 X^{4}+18 X^{2}-1$

$X_{7}=64 X^{7}-112 X^{5}+56 X^{3}-7 X$

$X_{8}=128 X^{8}-256 X^{6}+160 X^{4}-32 X^{2}+1$

$X_{9}=256 X^{9}-576 X^{7}+432 X^{5}-120 X^{3}+9 X$

$X_{10}=512 X^{10}-1280 X^{8}+1120 X^{6}-400 X^{4}+50 X^{2}-1$

$Y_{0}=1$

$Y_{1}=Y$

$Y_{2}=2 Y^{2}-1$

$Y_{3}=4 Y^{3}-3 Y$

$Y_{4}=8 Y^{4}-8 Y^{2}+1$

$Y_{5}=16 Y^{5}-20 Y^{3}+5 Y$

$Y_{6}=32 Y^{6}-48 Y^{4}+18 Y^{2}-1$

$Y_{7}=64 Y^{7}-112 Y^{5}+56 Y^{3}-7 Y$

$Y_{8}=128 Y^{8}-256 Y^{6}+160 Y^{4}-32 Y^{2}+1$

$Y_{9}=256 Y^{9}-576 Y^{7}+432 Y^{5}-120 Y^{3}+9 Y$

$Y_{10}=512 Y^{10}-1280 Y^{8}+1120 Y^{6}-400 Y^{4}+50 Y^{2}-1$ 


$$
\begin{aligned}
U & =C_{1}+C_{2} X_{1}+C_{3} Y_{1}+C_{4} X_{2}+C_{5} X_{1} Y_{1}+C_{6} Y_{2}+C_{7} X_{3} \\
& +C_{8} X_{2} Y_{1}+C_{9} X_{1} Y_{2}+C_{10} Y_{3}+C_{11} X_{4}+C_{12} X_{3} Y_{1} \\
& +C_{13} X_{2} Y_{2}+C_{14} X_{1} Y_{3}+C_{15} Y_{4}+C_{16} X_{5}+C_{17} X_{4} Y_{1} \\
& +C_{18} X_{3} Y_{2}+C_{19} X_{2} Y_{3}+C_{20} X_{1} Y_{4}+C_{21} Y_{5}+C_{22} X_{6} \\
& +C_{23} X_{5} Y_{1}+C_{24} X_{4} Y_{2}+C_{25} X_{3} Y_{3}+C_{26} X_{2} Y_{4} \\
& +C_{27} X_{1} Y_{5}+C_{28} Y_{6}+C_{29} X_{7}+C_{30} X_{6} Y_{1}+C_{31} X_{5} Y_{2} \\
& +C_{32} X_{4} Y_{3}+C_{33} X_{3} Y_{4}+C_{34} X_{2} Y_{5}+C_{35} X_{1} Y_{6} \\
& +C_{36} Y_{7}+C_{37} X_{8}+C_{38} X_{7} Y_{1}+C_{39} X_{6} Y_{2}+C_{40} X_{5} Y_{3} \\
& +C_{41} X_{4} Y_{4}+C_{42} X_{3} Y_{5}+C_{43} X_{2} Y_{6}+C_{44} X_{1} Y_{7} \\
& +C_{45} Y_{8}+C_{46} X_{9}+C_{47} X_{8} Y_{1}+C_{48} X_{7} Y_{2}+C_{49} X_{6} Y_{3} \\
& +C_{50} X_{5} Y_{4}+C_{51} X_{4} Y_{5}+C_{52} X_{3} Y_{6}+C_{53} X_{2} Y_{7} \\
& +C_{54} X_{1} Y_{8}+C_{55} Y_{9}+C_{56} X_{10}+C_{57} X_{9} Y_{1}+C_{58} X_{8} Y_{2} \\
& +C_{59} X_{7} Y_{3}+C_{60} X_{6} Y_{4}+C_{61} X_{5} Y_{5}+C_{62} X_{4} Y_{6} \\
& +C_{63} X_{3} Y_{7}+C_{64} X_{2} Y_{8}+C_{65} X_{1} Y_{9}+C_{66} Y_{10}
\end{aligned}
$$

$T_{i, j} \quad$ calculated temp. at $i$-th isochore and $j$-th isentrope

$P_{i, j} \quad$ calculated pressure at $i$-th isochore and $j$-th isentrope

$T_{i, j}{ }^{\mathrm{PR}}$ temp. at P-R $i$-th isochore and P-R $j$-th isentrope

$P_{i, j}{ }^{\mathrm{PR}}$ pressure at $\mathrm{P}-\mathrm{R} i$-th isochore and $\mathrm{P}-\mathrm{R} j$-th isentrope

$U \quad$ speed of sound at $T_{i, j}$ and $P_{i, j}$

$C_{i} \quad$ coefficients of the Chebyshev polynomial

\section{References:}

[1] M. Bijedić, N. Neimarlija, "Thermodynamic Properties of Gases from Speed-of-Sound Measurements," Int. J. Thermophys., 28, 268-278, 2007.

[2] M. Bijedić, N. Neimarlija, "Thermodynamic Properties of Carbon Dioxide Derived from the Speed of Sound," $J$. Iran. Chem. Soc., 5, 286-295, 2008.

[3] Estrada-Alexanders, A. F. (1996). Thermodynamic Properties of Gases from Measurements of the Speed of Sound (Doctoral dissertation), Imperial College, London, UK.

[4] M. Bijedić, N. Neimarlija, "Speed of Sound as a Source of Accurate Thermodynamic Properties of Gases," Lat. Am. Appl. Res., 43, 393-398, 2013.

[5] M. Bijedić, S. Begić, "Thermodynamic Properties of Vapors from Speed of Sound," J. Thermodyn., 2014, 1-5, 2014.

[6] M. Bijedić, N. Neimarlija, "Thermodynamic Properties of Liquids from Speed of Sound Measurements," Int. J. Thermodyn., 15, 61-68, 2012.

[7] M. Bijedić, S. Begić, "Density and Heat Capacity of Liquids from Speed of Sound," J. Thermodyn., 2016, 18, 2016.

[8] A. F. Estrada-Alexanders, Justo, D., "New Method for Deriving Accurate Thermodynamic Properties from Speed-of-Sound," J. Chem. Thermodyn., 36, 419-429, 2004.

[9] M. Bijedić, S. Begić, "Solution of the Adiabatic Sound Wave Equation as a Nonlinear Least Squares Problem," Int. J. Thermophys., 40, 1-36, 2019.

[10] M. J. Moran, H. N. Shapiro, Fundamentals of Engineering Thermodynamics, $5^{\text {th }}$ Ed. Chichester: John Wiley \& Sons, 2006.
[11] W. Chenney, D. Kincaid, Numerical Mathematics and Computing, $6^{\text {th }} E d$. Belmont: Thomson Brooks/Cole, 2008.

[12] C. de Boor, A Practical Guide to Splines. New York: Springer, 1978.

[13] G. H. Golub, C. F. Van Loan, Matrix Computations. Baltimore: Johns Hopkins University Press, 1983.

[14] T. E. Hull, W. H. Enright, and K. R. Jackson, User's Guide for DVERK - A Subroutine for Solving Non-Stiff ODEs. University of Toronto, 1976.

[15] K. Levenberg, "A Method for the Solution of Certain Non-Linear Problems in Least Squares," Q. Appl. Math., 2, 164-168, 1944.

[16] D. Marquardt, "An Algorithm for Least-Squares Estimation of Nonlinear Parameters," SIAM J. Appl. Math., 11, 431-441, 1963.

[17] J.J. Moré, B.S. Garbow, and K.E. Hillstrom, User Guide for MINPACK-1. Argonne National Laboratory Report ANL-80-74, 1980.

[18] C. Tegeler, R. Span, and W. Wagner, “A New Equation of State for Argon Covering the Fluid Region for Temperature from the Melting Line to $700 \mathrm{~K}$ at Pressures up to $1000 \mathrm{MPa}$, , J. Phys. Chem. Ref. Data, 28, 779-850, 1999.

[19] R. Span, E. W. Lemmon, R. T. Jacobsen, W. Wagner, and A. Yokozeki, "A Reference Equation of State for the Thermodynamic Properties of Nitrogen Covering the Fluid Region for Temperatures from 63.151 to $1000 \mathrm{~K}$ and Pressures to $2200 \mathrm{MPa}$," J. Phys. Chem. Ref. Data, 29, 1361-1433, 2000.

[20] R. Schmidt, W. Wagner, “A New Form of the Equation of State for Pure Substances and Its Application to Oxygen," Fluid Phase Equilibria, 19, 175-200, 1985.

[21] R. B. Stewart, R. T. Jacobsen, and W. Wagner, "Thermodynamic Properties of Oxygen from the Triple Point to $300 \mathrm{~K}$ with Pressures to $80 \mathrm{MPa}$," J. Phys. Chem. Ref. Data, 20, 917-1021, 1991.

[22] U. Setzmann, W. Wagner, “A New Equation of State and Tables of Thermodynamic Properties for Methane Covering the Range from the Melting Line to $625 \mathrm{~K}$ and Pressures up to $1000 \mathrm{MPa}$," J. Phys. Chem. Ref. Data, 20, 1061-1125, 1991.

[23] R. Span, W. Wagner, "A New Equation of State for Carbon Dioxide Covering the Fluid Region for Temperature from the Triple-Point Temperature to 1100 $\mathrm{K}$ at Pressures up to $800 \mathrm{MPa}$," J. Phys. Chem. Ref. Data, 25, 1509-1596, 1996.

[24] W. Wagner, A. Pruss, "The IAPWS Formulation 1995 for the Thermodynamic Properties of Ordinary Water Substance for General and Scientific Use," J. Phys. Chem. Ref. Data, 31, 387-535, 2002.

[25] D. Y. Peng, D. B. Robinson, "A New Two-Constant Equation of State," Ind. Eng. Chem. Fund., 15, 59-64, 1976.

[26] S. Lago, P. A. Giuliano Albo, "A Recursive Equation Method for the Determination of Density and Heat Capacity: Comparison Between Isentropic and Isothermal Integration Paths," J. Chem. Thermodyn., 42, 462-465, 2010. 